\title{
Flexo-electricity and the polarity of complex ferroelastic twin patterns
}

\author{
Ekhard K. H. Salje ${ }^{1,2, *}$, Suzhi $\mathrm{Li}^{3}$, Massimiliano Stengel ${ }^{4,5}$, \\ Peter Gumbsch ${ }^{6,7}$ and Xiangdong Ding ${ }^{1}$ \\ ${ }^{1}$ State Key Laboratory for Mechanical Behavior of Materials, Xi'an Jiaotong University, Xi'an \\ 710049, China \\ ${ }^{2}$ Department of Earth Sciences, University of Cambridge, Cambridge CB2 3EQ, UK \\ ${ }^{3}$ Institute of Nanotechnology, Karlsruhe Institute of Technology, 76344 Eggenstein- \\ Leopoldshafen, Germany \\ ${ }^{4}$ Institut de Ciencia de Materials de Barcelona (ICMAB-CSIC), Campus UAB, 08193 Bellaterra \\ (Barcelona), Spain \\ ${ }^{5}$ ICREA - Institució Catalana de Recerca i Estudis Avançats, 08010 Barcelona, Spain \\ ${ }^{6}$ Institute for Applied Materials, Karlsruhe Institute of Technology, 76131 Karlsruhe, Germany \\ ${ }^{7}$ Fraunhofer Institute for Mechanics of Materials IWM, 79108 Freiburg, Germany \\ * corresponding author, e-mail ekhard@esc.cam.ac.uk
}

\begin{abstract}
We study, by means of an atomistic toy model, the interplay of ferroelastic twin patterns and electrical polarization. Our molecular dynamics simulations reproduce polarity in straight twin walls as observed experimentally. We show, by making contact with continuum theory, that the effect is governed by linear flexoelectricity. Complex twin patterns, with very high densities of kinks and/or junctions, produce winding structures in the dipolar field, which are reminiscent of polarization vortices. By means of a "cold shearing" technique, we produce patches with high vortex densities; these unexpectedly show a net macroscopic polarization even if neither the original sample nor the applied mechanical perturbation breaks inversion symmetry by itself. These results may explain some puzzling experimental observations of "parasitic" polarity in the paraelectric phase of $\mathrm{BaTiO}_{3}$ and $\mathrm{LaAlO}_{3}$.
\end{abstract}




\section{Introduction}

Domain walls in ferroic materials are characterized by unique structural and electronic properties that markedly depart from those of the homogeneous crystalline phase. These often enable new functionalities that are forbidden by symmetry in the bulk, and are of interest for applications, e.g., in nanoelectronics ${ }^{1}$. Notable examples include the local emergence of ferromagnetic order in an otherwise antiferromagnetic material ${ }^{2}$, or the discovery of polarity ${ }^{3}$ at structural domain boundaries. The prime manifestation of this latter effect is $\mathrm{CaTiO}_{3}$, where twin-wall polarity was predicted $^{4}$ several years before it was observed experimentally ${ }^{5}$. The physical effect was formulated even earlier ${ }^{6,7}$, and relies on the repulsive biquadratic coupling between polarization and antiferrodistortive (AFD) tilts of the oxygen octahedral network. Octahedral distortions are large within domains, and suppress the tendency of the crystal to off-center therein; at twin boundaries, however, one of the AFD order parameters vanishes, allowing the latent polar instability to condense ${ }^{8,9}$. Similar effects were reported at ferroelectric ${ }^{10,11}$ and ferroelastic ${ }^{12}$ domain walls, which points to a rather ubiquitous impact of higher-order couplings on the physics of multiferroic materials ${ }^{8,13-20}$.

Meanwhile, in addition to the aforementioned biquadratic couplings, other mechanisms exist that can induce an electrical polarization at a ferroic domain boundary. At the interface between two neighboring domains the main order parameter undergoes a drastic variation on a short length scale, which can be as small as few unit cells. This implies that gradient effects, such as linear flexoelectricity, can locally induce a large polarization. Note that this is a universal effect, i.e. unlike the biquadratic coupling discussed above it does not require the material to have an unstable polar mode in its high-symmetry structure. Moreover, gradient terms typically break inversion symmetry, and therefore favor a specific direction of the polarization vector, $P$. (In the biquadratic case, the two ground states for $P$, namely "up" and "down" along an appropriate trajectory, have the same probability because $P$ enters the Hamiltonian only at even orders.) Both properties are appealing for applications, as they enable, in principle, a remarkable control of the polar degrees of freedom via ferroelastic domain engineering in a very broad range of candidate materials.

Such a control alone, however, does not guarantee that ferroelastic domain walls will automatically produce a useful functionality (e.g. a measurable electromechanical response) at the macroscopic scale. In simple stripe domain patterns, for example, neighboring boundaries are, by symmetry, associated with an opposite dipole moment, in such a way that their overall contribution cancels out. A possible way around consists in using complex twinned structures in order to avoid such a cancellation; yet, the two most densely twinned archetypal ferroelastics, namely the palmierite structure $\mathrm{Pb}_{3}\left(\mathrm{PO}_{4}\right)_{2}{ }^{21-23}$ and leucite ${ }^{24-26}$, show no measurable resonant polarity nor any pyroelectric effect. The central issue is hence to analyze under which circumstances can we expect polarity or ferroelectricity, and possibly extract some useful 
guidelines on how to realize such a condition in a real sample. This quest bears some conceptual relationships to the work on strong 'parasitic' polarity and even ferroelectricity in nominally paraelectric $\mathrm{BaTiO}_{3}{ }^{27,28}$ and related materials ${ }^{29,30}$. A similar macroscopic breakdown of inversion symmetry (and hence a measurable pyrocurrent) was demonstrated in paraelectric $(\mathrm{Ba}, \mathrm{Sr}) \mathrm{TiO}_{3}$ samples, which should be nominally nonpolar ${ }^{31}$. This unexpected effect was attributed to compositional gradients, which were inadvertently generated during processing.

Here we demonstrate, by means of molecular dynamics (MD) simulations of a simple toy model, that a qualitatively similar electromechanical response can be engineered by nonchemical means, via a careful control of the ferroelastic domain structure. In particular, we construct a twodimensional square lattice with two basis atoms (A and B) carrying an opposite charge, whose mutual coupling is governed by Coulomb interactions and short-range central forces. The latter are such that the A lattice drives a ferroelastic transition at $70 \mathrm{~K}$, while the B lattice is purely harmonic and responds to heterogeneous deformations with a net dipole moment. For an isolated planar domain we show, after establishing a rigorous link between the discrete lattice model and the continuum framework via a long-wave procedure, that the model accurately reproduces the analytic solution of the flexoelectric free energy functional proposed in Ref. ${ }^{32}$. The advantage of the present model is that it allows us to explore complex domain configurations, which are unaccessible analytically. We find that twinned domain structures contain a large number of kinks and vortices and generally display a net macroscopic polarization. Our results may explain the surprising experimental finding of polarity in fine-grained materials whose bulk crystal structure is centrosymmetric ${ }^{27-31,33}$. More generally, this work opens exciting new avenues in the design of low-cost electromechanical devices with tailored properties.

\section{Atomistic model}

The standard ferroelastic model was first introduced ${ }^{34}$ for MD for complex ferroelastic domain patterns with many particles (e.g., $10^{6}$ atoms) and long MD running times (e.g., $10^{4}$ phonon times). We refer the reader to this paper for the details of the simulation. The model is based on simple nonlinear elastic interactions (Landau springs) and harmonically coupled displacements of charged particles of the A sublattice. To study polarity, the potential is modified as follows. The configuration of the cation A sublattice is kept as in ref. ${ }^{34}$, except for a weak additional $4^{\text {th }}$ neighbor interaction, which is used here to calibrate the energy of twin boundaries. The interactions within the anion B sublattice consist of harmonic springs between nearest-neighbors and second nearest-neighbors. The two sublattices are coupled via a weak harmonic spring and via Coulomb forces. (The springs are necessary to avoid the collapse of the B sublattice onto the A sublattice.) This new potential generates a ferroeleastic transition at $70 \mathrm{~K}$. All model parameters are summarized in Table 1. The model is constructed such that the equilibrium single crystal (embedded in a 3-dimensional matrix) undergoes a tetragonal $4 / \mathrm{mmm}$ to orthorhombic 
$\mathrm{mmm}$ phase transition. Both phases are centrosymmetric, as there is no ferroelectric instability in the system (see supplementary Fig. S1). The symmetry is broken inside twin walls to noncentrosymmetric $\mathrm{m}$, while an additional symmetry breaking to 1 can occur within vortex structures ${ }^{35,36}$. The bulk is purely ferroelastic, while the domain boundaries are polar. The model parameters are inspired by $\mathrm{SrTiO}_{3}$ with the energy scale determined by $T_{\mathrm{c}}=105 \mathrm{~K}$, a typical ferroelastic shear angle of $2^{\circ}$, and wall dipoles related to displacements of $6 \mathrm{pm}$ as observed in $\mathrm{CaTiO}_{3}$. All simulations were performed using the LAMMPS code ${ }^{37}$. The temperature of the sample was held constant by the Nosé-Hoover thermostat ${ }^{38,39}$. Periodic boundary conditions were adopted to avoid surface effects.

To start with, we examine the impact of twin boundaries on the relaxed atomic structure (Fig. 1). The initial configuration is a sandwich with two preexisting horizontal twin boundaries. The system size is $10 \mathrm{~nm} \times 10 \mathrm{~nm}$. The left and right columns in Fig. 1 show the atomic images for both $\mathrm{A}$ and $\mathrm{B}$ sublattices, respectively. The color is mapped according to atomic shear strain $\left(\tau_{\mathrm{xy}}\right)$. The middle column in Fig. 1 shows the dipole configuration near twin boundaries at $y=2.5$ $\mathrm{nm}$. The twin boundary is clearly polar near the center of the twin wall.

To qualitatively understand the microscopic origin of the calculated behavior, it is useful to analyze the relaxed configuration in terms of two sublattice-specific "strain" fields (defined by the relative displacements of either atoms A or B), and a polarization field (defined by the local offset between the A and B sublattices). Figure 2 shows the resulting profiles of strain and induced polarization. The anharmonic potential of the A sublattice generates the twin boundary while the harmonic B sublattice relaxes onto the template of the A sublattice. The strain profile is complex because the "internal stiffness" of the A sublattice is much greater than that of the B sublattice. As a consequence, the A sublattice generates an abrupt strain discontinuity at the twin boundary whereas the B sublattice produces a smooth transition profile. The wall thicknesses $w$ were then determined by fitting a tanh-profile to the simulated wall profiles: $w_{\mathrm{A}}=0.01 \mathrm{~nm}$ for the A sublattice and $w_{\mathrm{B}}=0.134 \mathrm{~nm}$ for the B sublattice (see supplementary Fig. S2). This tanhprofile fits very well the "soft" B lattice but not the stiff A lattice where the role of higher order couplings lead to ripples (upturns of the order parameter near the wall), which have been discussed in detail in refs. ${ }^{32,40,41}$. The local shift between the two sublattices generates the dipole polarization density $P$ and follows well the predictions of Refs. ${ }^{7,13,41}$. We shall discuss the physical origin of these effects in Section III.

While the individual walls are polar, the overall macroscopic polarization of the system (cell average) vanishes. To prove this, we simulate larger time intervals and rely on the ergodicity of the pattern formation; the macroscopic $P$ is then defined as its time average during relaxation. In specific, we relax the simple twin pattern for $1.5 \mathrm{~ns}$ and take the snapshots every $1 \mathrm{ps}$. We then determine the averaged polarization $\langle P\rangle=q\langle s\rangle / V$, where $q$ is the electron charge, $\langle s\rangle$ (= $\left.\sqrt{\left\langle s_{\mathrm{x}}\right\rangle^{2}+\left\langle s_{\mathrm{y}}\right\rangle^{2}}\right)$ is the averaged polarization displacement, and $V$ is the system volume. 
Figure S3a shows the variation of $\left\langle s_{\mathrm{x}}\right\rangle$ and $\left\langle s_{\mathrm{y}}\right\rangle$ as the function of relaxation time. We can see that the $\left\langle s_{\mathrm{x}}\right\rangle$ and $\left\langle s_{\mathrm{y}}\right\rangle$ eventually oscillate around zero. Figure S3b shows the variation of $\langle P\rangle$ as the function of relaxation time. Wall related polarization decays quickly to zero. Each wall has a polarization towards the apex of the twin boundary and each twin boundary has a second twin boundary with opposite direction of the apex. This means that the polarizations of a multitude of twin walls compensate each other to that the resulting $\langle P\rangle$ becomes zero. This is physically due to the fact that a stripe domain pattern globally preserves inversion symmetry, even if the individual boundaries do not. To achieve a nonvanishing value of $\langle P\rangle$, a more complex domain configuration is needed, as we shall see in Section IV.

\section{Continuum analysis}

To rationalize the physics that leads to the peculiar relaxation patterns discussed above in terms of macroscopic concepts, we shall make contact with the continuums theory proposed in Ref. ${ }^{32}$. The state of the atomic MD system can be described by two vector fields, $\mathbf{u}_{\mathrm{A}}$ and $\mathbf{u}_{\mathrm{B}}$, for the (local) displacement of sublattices A and B from their high-symmetry position. By assuming that the perturbations (distortions) are smooth on the scale of the interatomic distances, we can treat both $\mathbf{u}_{\mathrm{A}}$ and $\mathbf{u}_{\mathrm{B}}$ as continuum fields. We define, by a unitary transformation, two auxiliary fields that we call, respectively, displacement (u) and polarization (P) field. While the displacement (acoustic branch) at the zone center is always a rigid translation of lattices, there is a degree of freedom in choosing the microscopic nature of the polarization (optical branch). If we were to use the normal modes of the system as a reference then the optical mode would either look like an antiphase excitation of the two lattices with equal amplitude, or more like an oscillation of the lighter sublattice while the heavier one remains more or less constant - depending on the A/B mass ratio. Using phonons is not strictly necessary though: in an energy functional one only needs to ensure that the change of coordinates is made via a nonsingular transformation, and otherwise seek the choice that yields the simplest physical description. After observing that sublattice $\mathrm{B}$ is purely harmonic in the simulations, it is therefore most convenient to write $\mathbf{u}_{\mathrm{A}}=$ $\mathbf{u}, \mathbf{u}_{\mathrm{B}}=\mathbf{u}+\mathbf{P}$. This implies identifying, once and for all, the deformations with the sublattice A (which is natural, as it provides the ferroelastic behavior), and yields a polarization that is correctly written as the difference of the two sublattice shifts. We can then construct a Gibbs free energy (1) for the simplest case when a shear strain wave couples with a transverse optical wave along the same direction:

$H(Q, P)=\mathrm{A} / 2 Q^{2}+\mathrm{B} / 4 Q^{4}+g_{\mathrm{Q}} / 2 Q^{, 2}+\chi^{-1} / 2 P^{2}+g_{\mathrm{P}} / 2 P^{, 2}-f P Q^{\prime}$

The first three terms consist in the well-known ferroelastic energy functional as a function of the strain $Q=u^{\prime}$ and strain gradient $Q^{\prime}=u^{\prime}$, where $\mathrm{A}, \mathrm{B}$ and $g_{\mathrm{Q}}$ are the corresponding coupling coefficients. Flexoelectricity enters the Hamiltonian via the flexocoupling coefficient, $f$. To 
reproduce the model results we also need the harmonic potential for the polarization, $\chi^{-1}$ (i.e. the inverse susceptibility), and its correlation coefficients, $g_{\mathrm{P}}$.

The value of coefficients in the ferroelastic part can be determined by the potential itself as $\mathrm{A}=$ $39.6 \mathrm{eV}, \mathrm{B}=32894.0 \mathrm{eV}$ and $g_{\mathrm{Q}}=5.6 \mathrm{eV}^{2}$ (see Appendix). The ground state of the system is uniformly sheared with $Q_{0}=\sqrt{-\mathrm{A} / \mathrm{B}}$. The characteristic length $\lambda=\sqrt{-g_{\mathrm{Q}} / \mathrm{A}}$ defines the width of the domain wall. Given the numerical value of the above coefficients, we calculate the characteristic length $\lambda=0.037 \mathrm{~nm}$, which is close to the value $0.01 \mathrm{~nm}$ of the A sublattice which we fitting in the tanh-profile above. We fit the value of $\chi^{-1}=0.0134 \mathrm{eV} /\left(\mathrm{C} / \mathrm{m}^{2}\right)^{2}$ and $g_{\mathrm{P}}=0.0468$ $\mathrm{eV} /\left(\mathrm{C} / \mathrm{m}^{2} / \AA\right)^{2}$ by using a frozen-phonon model (see Appendix).

To compute the flexocoupling coefficient, $f$, we first calculate the flexoelectric coefficient, $\mu$. To that end, we deform the A sublattice into a frozen sine-wave and relax the B sublattice to the energy minimum at $0.1 \mathrm{~K}$ (see supplementary Fig. S3). The modulation generates strain gradients, which, in turn, produce dipoles via the flexoelectric effect. These dipoles are in phase with the sine waves of the A and B sublattices. The correlation between the strain gradients and the dipole density is strictly linear while the correlation between the dipole density and the strains is circular (see supplementary Fig. S4). The bulk flexoelectric coefficient $(\mu)$ is defined as the slope of the polarization density with the shear strain gradient in the sine-wave model, which is obtained by fitting the data linearly with $\mu=3.57 \mathrm{C} / \mathrm{m}^{2} / \AA^{-1}$. The coefficient $f$ is then obtained to be $\mu / \chi=0.048 \mathrm{eV} /\left(\mathrm{C} / \mathrm{m}^{2} / \AA\right)$.

Further we can find an approximate solution for describing the distribution of polarization along the direction normal to domain wall as $P(y)$. We shall initially assume that the strain gradient field at a domain wall is a Dirac delta in $y$ (which is reasonable as the length scale of $Q$ is much shorter than that of the polarization),

$Q^{\prime}(y)=2 Q_{0} \delta(y)$,

The governing equation for $P$ reads

$\chi^{-1} P-f Q^{\prime}-g P^{\prime \prime}=0$,

Solutions are of the type

$P(y)=P_{0} e^{-\frac{|y|}{\sigma}}$

where $\sigma=\sqrt{g / \chi^{-1}}$ is the correlation length for the polarization. We only need to determine the constant $P_{0}$. To this end, it suffices to integrate the equation above in a neighborhood of $y=0$. We have (after keeping only those terms that tend to a constant upon making this interval smaller),

$f Q_{0}+g P_{0}(+)=0$,

where $P_{0}(+)$ indicates the first derivative of $P$ on the positive $y$ side. We easily obtain 


$$
P_{0}=\frac{f Q_{0} \sigma}{g} .
$$

Given the numerical value of the above coefficients, we estimate the characteristic length, $\sigma=$ $0.19 \mathrm{~nm}$ and the polarization at the wall, $P_{0}=0.067 \mathrm{C} / \mathrm{m}^{2}$. The two values matches perfectly with fits of the decay of $P$ away from the polar domain wall (Fig. $2 \mathrm{~b}$ ) with $0.19 \mathrm{~nm}$ and $0.068 \mathrm{C} / \mathrm{m}^{2}$, respectively, as shown in Fig. 4.

It is interesting to compute the total polarization that is generated at the ferroelastic wall. This is

$$
P^{\mathrm{tot}}=2 \int_{0}^{+\infty} P(y) d y \text {, }
$$

This is easily computed and yields

$$
P^{\mathrm{tot}}=2 P_{0} \sigma=2 \frac{f Q_{0} \sigma}{g}=2 f Q_{0} \chi \text {. }
$$

This provides us with an important result: while the details of how the polarization distributes itself around the wall depend on the correlation coefficient of $P$, the total polarization is completely independent of $g$. This, in particular, is trivially given by the flexoelectric coefficient $\mu=f \chi$, multiplied by the total strain difference between the two domains $Q=2 Q_{0}$.

This analysis provides us with two important pieces of information: (i) the polarity of the twin walls that we described in the previous Section is entirely due to the linear flexoelectric effect; (ii) the "nonlocal" nature of the polarization pattern, which propagates several unit cells away from the wall, is due to the correlation coefficient, $\mathrm{g}_{\mathrm{P}}$. It is important to note, in this context, that while the decay length directly depends on $g_{P}$, the total dipole moment per unit area of the wall (i.e. the polarization integrated along the normal to the wall plane) is independent of it, and is very accurately given by $P^{\text {tot }}=2 Q_{0} \mu$. For this reason a local analysis (polarization versus straingradient amplitude at a given point) is inappropriate here: the structural distortions that are responsible for the polar displacements are confined to sharp boundaries with essentially zero thickness, whereas the polarization field propagates deep into the domains, where the ferroelastic strain is essentially uniform. Such a complication, of course, adds up to other sources of nonlocality that are proper of more conventional flexoelectric phenomena, e.g. long-range electrostatic interactions, calling for special care in the interpretation of the results.

\section{Complex patterns}

Complex patterns are generated by "cold shearing" techniques ${ }^{42}$. In single stress geometry only horizontal twins are generated but no vertical twins. The high twin density leads to kinks inside the twin walls, as shown in Fig. 5a-c, where only a small region ( $4 \mathrm{~nm} \times 3 \mathrm{~nm}$ ) out of a larger sample $(10 \mathrm{~nm} \times 10 \mathrm{~nm})$ is displayed. The kinks generate additional features in the polarization patterns (Fig. 5d) that were not present in the earlier examples. For example, the polarization 
locally acquires nonvanishing vertical components, which are forbidden by symmetry in the previously considered case of a straight domain boundary. Moreover, the interaction between neighboring walls gives rise to characteristic winding patterns, reminiscent of a vortex structure. These new features could be, in principle, determined by long-range electrostatic interactions. Previously, polarization vortices near domain walls have been shown to form in ferroelectric closure domains under electric fields ${ }^{43}$. The inevitable conclusion is that the new features are, as before, induced via the flexoelectric effect, with the only difference that the increased complexity in the deformation field now activates some components of the strain-gradient tensor that were formerly inactive. For instance, flexocouplings associated with gradients of the diagonal components of the strain tensor can no longer be neglected, contrary to the flat twin wall case where only the shear strain $\left(\varepsilon_{\mathrm{xy}}\right)$ was present. The color map of $\varepsilon_{\mathrm{xx}}$ and $\varepsilon_{\mathrm{yy}}$ indeed confirms that these components are unavoidable in a vicinity of a kink. (From the point of view of elastic theory, a kink bears some similarities to an edge dislocation.) Also, the shear strain now displays strong gradients along the horizontal direction as well, which may also contribute to the normal (vertical) components of $P$.

Domain patterns with even greater complexity were generated with shear in two orthogonal directions (horizontal and vertical). The predominant new features in this configuration are junctions and right angle corners between twin boundaries in different directions ${ }^{34,43,44}$. The junction density has previously been used as measure for the complexity of a domain pattern ${ }^{42}$. The resulting junction densities in these patterns (Fig. 6a-c) are almost as high as in conventional tweed structures ${ }^{45,46}$. As in the previous case, the local strain in the junction regions is no longer a simple shear but includes a component of dilatory strain along the horizontal and vertical axes; the magnitude of $\varepsilon_{\mathrm{xx}}$ and $\varepsilon_{\mathrm{yy}}$ is even larger here than in a vicinity of a kink, indicating that the junctions entail a considerable elastic mismatch, and are therefore energetically very expensive. The resulting polarization pattern displays a correspondingly higher degree of complexity (Fig. 6d), with a marked occurrence of vortex-like patterns. We stress that these structures should not be thought as resulting from electrostatic flux closure, though. To prove this point, we have performed a computational experiment where we switched off the Coulomb interactions in our model, and relaxed again the B lattice by keeping the ferroelastic A lattice fixed to the same configuration as in Fig. 6. The resulting polarization field was essentially unaltered, except for a slight uniform decrease in the amplitude (see supplementary Fig. S5). Thus, flexoelectricity alone, together with the correlation term (which is responsible for the spatially nonlocal relationship between elastic and polar fields), is responsible for the patterns of Fig. 6d, which points to a remarkable conclusion: polarization vortices can emerge from a complex ferroelastic structure via linear flexoelectricity alone.

We finally simulate the averaged polarization of the whole sample $(10 \mathrm{~nm} \times 10 \mathrm{~nm})$. As we showed earlier, a simple twin pattern generates a vanishing $\langle P\rangle$ (the brackets indicate averaging over a long relaxation time). However, kinks and junctions can break the macroscopic inversion 
symmetry of the sample and allow for a net polarity (and therefore pyroelectricity). Here we took four randomly generated complex twin patterns with vortices (see Fig. 7a-d). Figure 7e shows the variation of $\langle P\rangle$ as the function of relaxation time. One can clearly see that, after some fluctuation in the early stage, $\langle P\rangle$ eventually converges to a finite value (of the order of $10^{-2} \sim$ $10^{-1} \mathrm{mC} / \mathrm{m}^{2}$ ) in all four cases. It may appear surprising that polarity emerges from a nonpolar macroscopic sample undergoing a nonpolar perturbation. The point is that, because of thermal fluctuations, symmetry-breaking defects in the ferroelastic structure may nucleate at some random location in the sample, and subsequently act as a bias field for propagation of the domain wall lines and further nucleation of adjacent defects. This idea bears some similarities to the Garten-Trolier-McKinstry model ${ }^{28}$, where some inherent polar instability is activated by the flexoelectric effect. In our case, the strain field plays the role of the primary order parameter, and flexoelectricity translates the macroscopic asymmetry in the ferroelastic structure into a net polarization.

\section{Discussion and conclusion}

Our results, showing that a macroscopic $P$ can emerge from a nonpolar solid via symmetrybreaking inhomogeneities and defects in the ferroelastic domain structure, may open exciting perspectives in designing multifunctional oxide materials with tailored properties. We believe that the practical applicability of such a strategy may depend on two main factors. The first issue is ensuring that a certain type of mechanical treatment (e.g. the cold shearing procedure described here) leads to a predictable polarity in a given macroscopic sample; the second is ensuring that the resulting polar state is stable under operation conditions (i.e. preventing the domain structure from switching back to a nonpolar state).

Regarding the first point, note that one can use a much wider class of mechanical perturbations to achieve the desired asymmetry in the ferroelastic pattern. An obvious possibility would be to cool down different parts of the sample at different rates; this means that a temperature gradient will be present when the system undergoes the ferroelastic transition, and this should guarantee a sizeable asymmetry (and hence, a polar state). This would be close in spirit to the work of Biancoli et al. ${ }^{31}$, where temperature gradients during sintering were deemed responsible for an intrinsic asymmetry of nominally paraelectric samples. Another option would be to mechanically process the sample after it has transitioned to a ferroelastic state, but this time by using a symmetry-breaking deformation. Bending the sample, for example, is known to induce ferroelastic domain-wall motion that contribute to the effective flexoelectric coefficient ${ }^{47}$. If the bending is done at a temperature reasonably close to $T_{C}$, domains should be easy to arrange into a polar state; subsequent cooling will freeze the domains but preserve polarity (and hence piezoelectricity) and all this in a material that is nominally nonpolar ${ }^{9}$. 
This latter observation brings us to our second point, concerning the stability of the "polar" ferroelastic pattern. In our model, we have considered two main sources of polarity, kinks and junctions. These, however, are highly energetic features, and may be hard to stabilize in an otherwise defected-free sample. To gain a flavor of how robust these patterns really are in the context of our toy model (see supplementary Fig. S6), we have followed on the computational experiment (recall that we suppressed the Coulomb interactions to test the physical origin of the vortex patterns), and proceeded to full relaxation of the A and B lattices (earlier we had kept the A lattice fixed, as we were primarily interested in the induced polarization). The ferroelastic structure significantly changed, with a marked decrease in the average polarity. This result confirms that these structures are indeed highly energetic, and therefore sensitive to small perturbations. The alternative procedures that we have discussed in the previous paragraph may lead to a more robust polar structure; verifying such a speculation is left to future studies.

\section{Achnowledgement}

EKHS is grateful to EPSRC for financial support (EP/K009702/1). SL and PG appreciate the support by Helmholtz Programme Science and Technology of Nanosystems (STN) (Vorhabensnumber 43.22.01). MS acknowledges support by MINECO-Spain through Grants No. FIS2013-48668-C2-2-P and No. SEV-2015-0496, and by Generalitat de Catalunya (2014 SGR301). X.D. appreciates the support of NSFC (51171140, 51231008, 51320105014, 51321003), the 973 Programs of China (2012CB619402), and 111 project (B06025). 


\section{References}

G. Catalan, J. Seidel, R. Ramesh, and J. F. Scott, Reviews of Modern Physics 84, 119 (2012).

M. Carpenter, E.K.H. Salje, and C. J. Howard, Physical Review B 85, 224430 (2012).

E. K. H. Salje, ChemPhysChem 11, 940 (2010).

L. Goncalves-Ferreira, S. A. Redfern, E. Artacho, and E.K.H. Salje, Physical review letters 101, 097602 (2008).

S. Van Aert, S. Turner, R. Delville, D. Schryvers, G. Van Tendeloo, and E.K.H. Salje, Advanced Materials 24, 523 (2012).

M. Daraktchiev, G. Catalan, and J. F. Scott, Physical Review B 81, 224118 (2010).

B. Houchmandzadeh, J. Lajzerowicz, and E. Salje, Journal of Physics: Condensed Matter 3, 5163 (1991).

J. Î́niguez, M. Stengel, S. Prosandeev, and L. Bellaiche, Physical Review B 90, 220103 (2014).

D. D. Viehland and E. K.H. Salje, Advances in physics 63, 267 (2014).

A. Lubk, M. Rossell, J. Seidel, Y. Chu, R. Ramesh, M. Hÿtch, and E. Snoeck, Nano letters 13, 1410 (2013).

J. Seidel, R. K. Vasudevan, and N. Valanoor, Advanced Electronic Materials 2 (2016).

E. K. H. Salje, Annual Review of Materials Research 42, 265 (2012).

S. Conti, S. Müller, A. Poliakovsky, and E.K.H. Salje, Journal of Physics: Condensed Matter 23, 142203 (2011).

H. Pöttker and E. K.H. Salje, Journal of Physics: Condensed Matter 26, 342201 (2014).

E. Salje, Physics and Chemistry of Minerals 12, 93 (1985).

E.K.H. Salje and M. Carpenter, Journal of Physics: Condensed Matter 23, 462202 (2011).

S. Farokhipoor, et al., Nature 515, 379 (2014).

D. Lee, H. Xu, V. Dierolf, V. Gopalan, and S. R. Phillpot, Applied Physics Letters 98, 092903 (2011).

A. Aird and E.K.H. Salje, The European Physical Journal B-Condensed Matter and Complex Systems 15, 205 (2000).

M. Calleja, M. T. Dove, and E. K.H. Salje, Journal of Physics: Condensed Matter 15, 2301 (2003).

E. K. H. Salje and B. Wruck, Physical Review B 28, 6510 (1983).

U. Bismayer and E. K. H. Salje, Acta Crystallographica Section A: Crystal Physics, Diffraction, Theoretical and General Crystallography 37, 145 (1981).

O. Aktas, E.K.H. Salje, and M. Carpenter, Journal of Physics: Condensed Matter 25, 465401 (2013).

D. C. Palmer, E.K.H. Salje, and W. W. Schmahl, Physics and Chemistry of Minerals 16, 714 (1989).

M. T. Dove, T. Cool, D. C. Palmer, A. Putnis, E.K.H. Salje, and B. Winkler, American Mineralogist 78, 486 (1993).

O. Aktas, M. A. Carpenter, and E.K.H. Salje, American Mineralogist 100, 2159 (2015).

A. Pramanick, X.P. Wang, C. Hoffmann, S. O. Diallo, M. R. V. Jørgensen, and X.-L. Wang, Physical Review B 92, 174103 (2015).

L. M. Garten and S. Trolier-McKinstry, Journal of Applied Physics 117, 094102 (2015). 
J. Narvaez, S. Saremi, J. Hong, M. Stengel, and G. Catalan, Physical review letters 115, 037601 (2015). O. Aktas, E.K.H. Salje, S. Crossley, G. I. Lampronti, R. W. Whatmore, N. D. Mathur, and M. A. Carpenter, Physical Review B 88, 174112 (2013).

A. Biancoli, C. M. Fancher, J. L. Jones, and D. Damjanovic, Nature materials 14, 224 (2015).

H. Pöttker and E.K.H. Salje, Journal of Physics: Condensed Matter, in press (2015).

E. K. H. Salje, M. Alexe, G. Nataf, S. Kustov, M. Weber, and J. Schiemer, submitted.

Z. Zhao, X. Ding, and E. Salje, Applied Physics Letters 105, 112906 (2014).

E.K.H. Salje and J. Scott, Applied Physics Letters 105, 252904 (2014).

T. Zykova-Timan and E.K.H. Salje, Applied Physics Letters 104, 082907 (2014).

S. Plimpton, Journal of computational physics 117, 1 (1995).

S. Nosé, The Journal of chemical physics 81, 511 (1984).

W. G. Hoover, Physical Review A 31, 1695 (1985).

B. Houchmanzadeh, J. Lajzerowicz, and E. Salje, Phase Transitions: A Multinational Journal 38, 77 (1992).

R. Ahluwalia, A. K. Tagantsev, P. Yudin, N. Setter, N. Ng, and D. J. Srolovitz, Physical Review B 89, 174105 (2014).

X. Ding, Z. Zhao, T. Lookman, A. Saxena, and E. K. H. Salje, Advanced Materials 24, 5385 (2012).

E.K.H. Salje, S. Li, Z. Zhao, P. Gumbsch, and X. Ding, Applied Physics Letters 106, 212907 (2015).

E. K. H. Salje and Y. Ishibashi, Journal of Physics: Condensed Matter 8, 8477 (1996).

E.K.H. Salje, American Mineralogist 100, 343 (2015).

E. Dul'kin, E.K.H. Salje, O. Aktas, R. W. Whatmore, and M. Roth, Applied Physics Letters 105, 212901 (2014).

P. Zubko, G. Catalan, P.R.L. Welche, A. Buckley, and J. Scott, Physical review letters 99, 167601 (2007). 
Table 1 The interaction potential for describing flexoelectric effect in binary system. For different range of nearest neighbor $(\mathrm{NN})$ interactions, we set different form of potential function to describe them.

\begin{tabular}{c|c|l}
\hline interactions & \multicolumn{1}{|c}{ range } & \multicolumn{1}{c}{ potential form } \\
\hline & 1 st NN & $20(\mathrm{r}-1)^{2}$ \\
& 2nd NN & $-10(\mathrm{r}-\sqrt{2})^{2}+8000(\mathrm{r}-\sqrt{ } 2)^{4}$ \\
A-A & 3rd NN & $8(\mathrm{r}-2)^{4}$ \\
& 4 th NN & $-10(\mathrm{r}-\sqrt{5})^{2}+5100(\mathrm{r}-\sqrt{5})^{4}$ \\
\hline \multirow{2}{*}{ B-B } & $1 \mathrm{st} N \mathrm{~N}$ & $20(\mathrm{r}-1)^{2}$ \\
& $2 \mathrm{nd} \mathrm{NN}$ & $1.5(\mathrm{r}-\sqrt{ } 2)^{2}$ \\
\hline A-B & $1 \mathrm{st} \mathrm{NN}$ & $0.5(\mathrm{r}-\sqrt{ } 2 / 2)^{2}$ \\
\hline
\end{tabular}



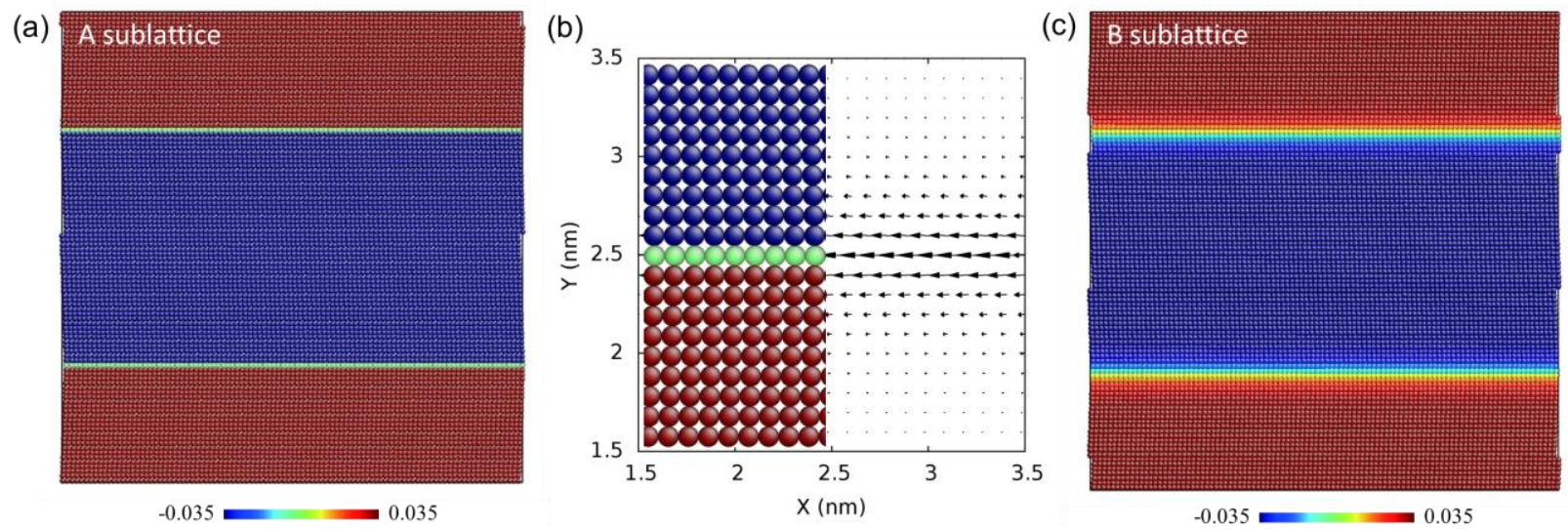

Figure 1 (a) Atomic images and (b) the dipoles near the twin boundaries for the A sublattice. (c) Atomic images near twin boundaries in B sublattice. The color shown in (a) and (c) represent the atomic shear strain $\left(\tau_{\mathrm{xy}}\right)$, see scale bar. Dipole displacements are amplified by a factor of 25 . 

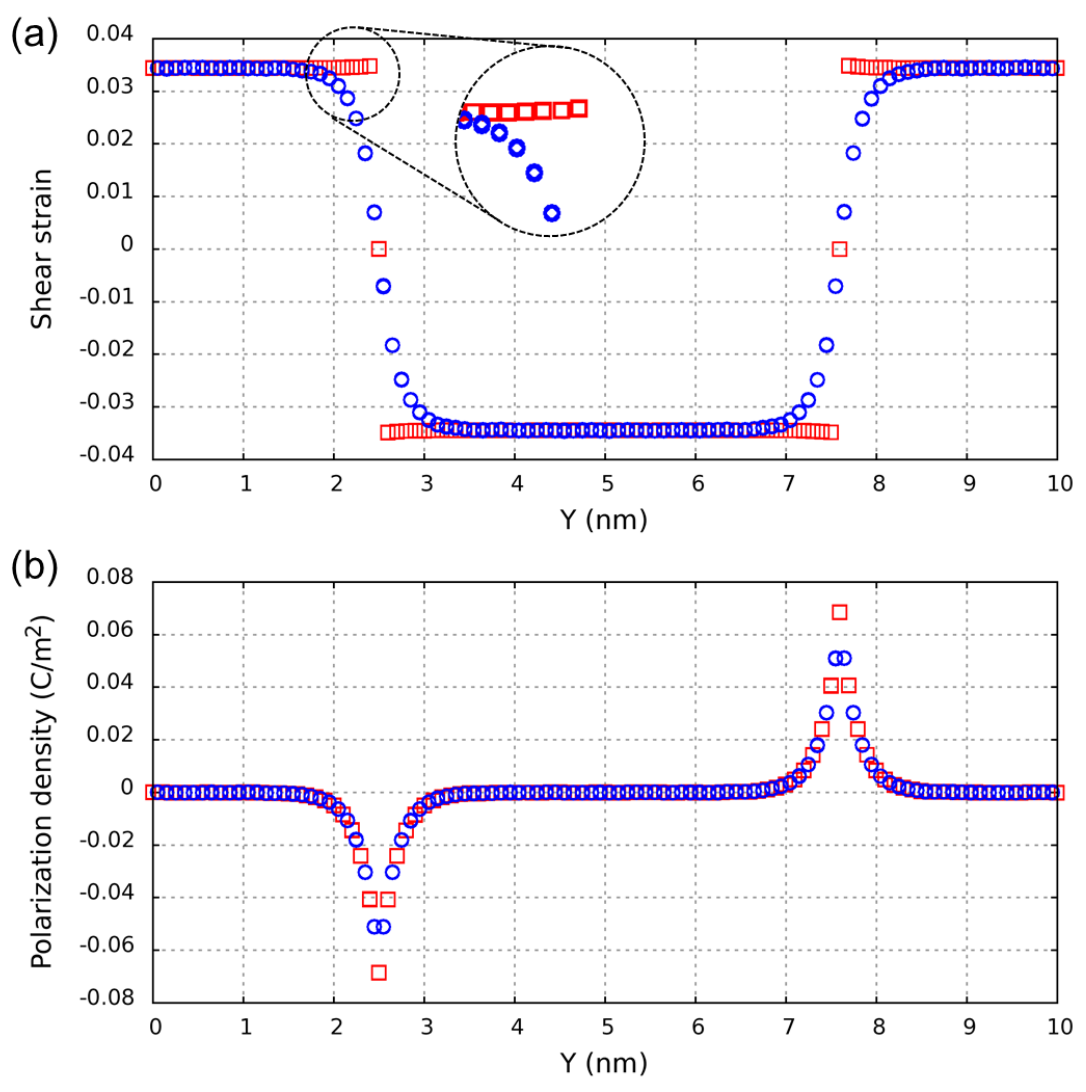

Figure 2 (a) Wall profiles of the A sublattice (red squares) and B sublattice (blue circles). The strains are shear strains for each sublattice. The stiff A sublattice has a sharp interface with a wall thickness of 0.01 $\mathrm{nm}$, the soft B lattice shows a much smoother interface with a thickness of $0.134 \mathrm{~nm}$. (b) Breather profiles of the induced polarization inside the ferroelastic walls. 

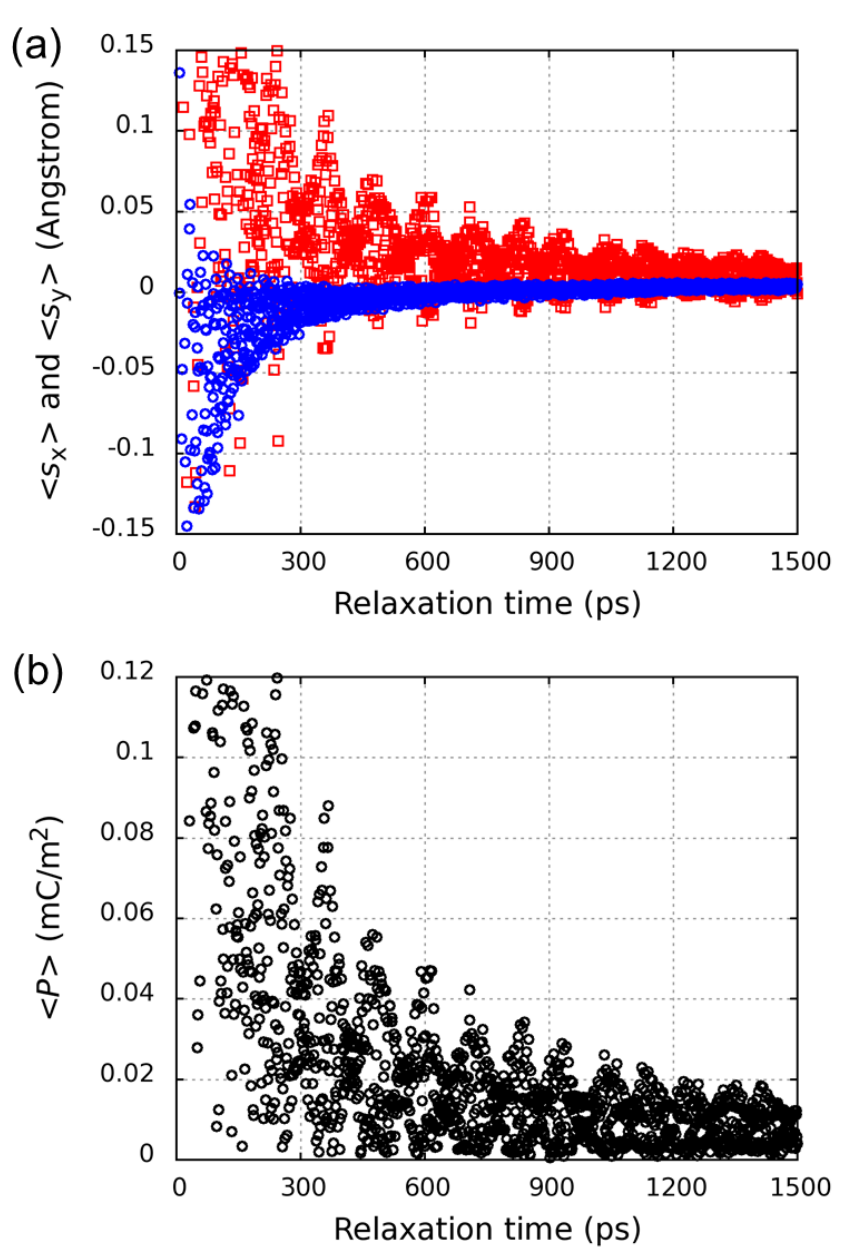

Figure 3 The variation of (a) $\left\langle s_{\mathrm{x}}\right\rangle$ (red squares) and $\left\langle s_{\mathrm{y}}\right\rangle$ (blue circles) and (b) $\langle P\rangle$ as the function of relaxation time. 


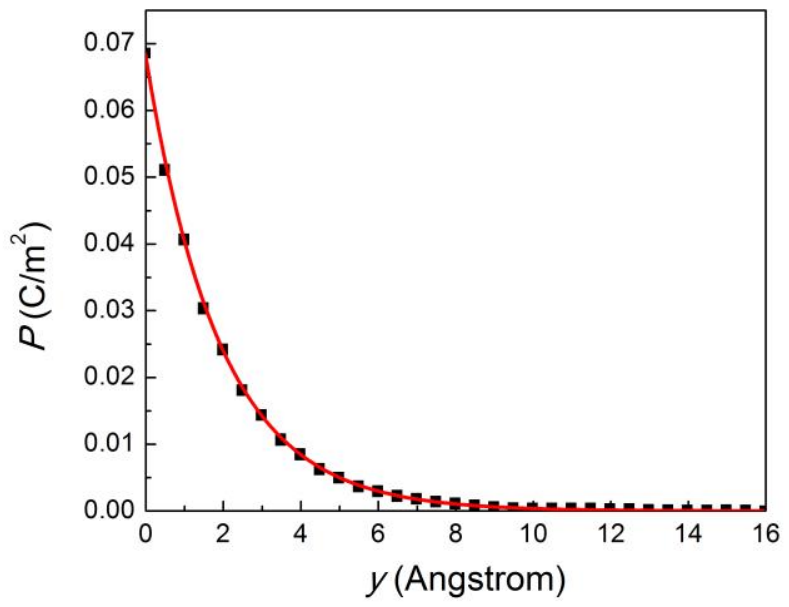

Figure 4 Fitting the decay of polarization along the direction normal to domain wall in an exponent form. The black data are extracted from atomistic simulation in Fig. $2 b$. The red line is the fitting curve. 

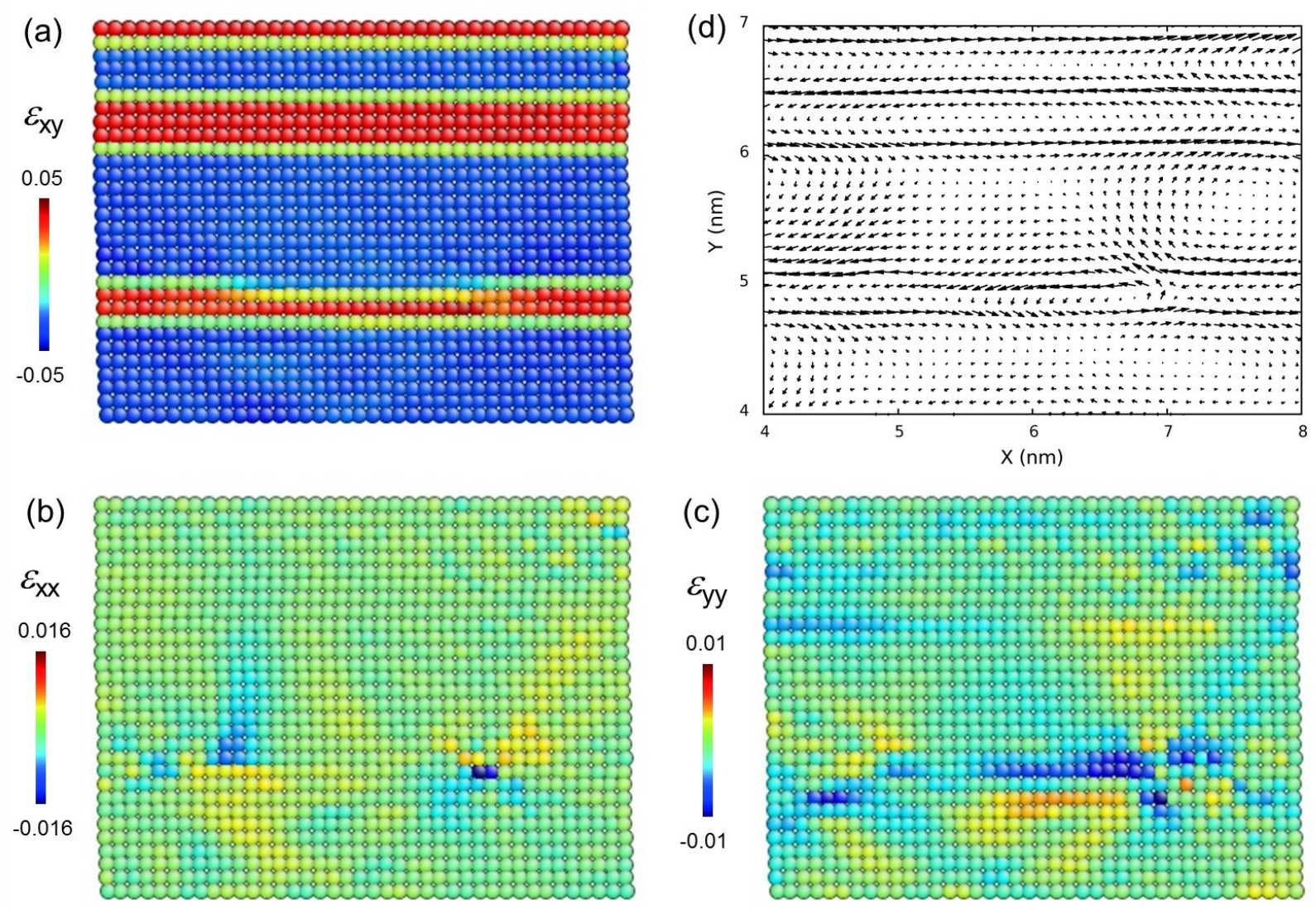

Figure 5 Sheared twin structure with multiple kinks inside the domain walls: (a)-(c) atomic images in view of $\varepsilon_{\mathrm{xy}}, \varepsilon_{\mathrm{xx}}$ and $\varepsilon_{\mathrm{yy}}$, respectively. (d) dipole configuration. Dipole displacements are amplified by a factor of 25. Each kink generates a polar vortex structure. These vortex structures do not follow the strain or strain gradient and add "noise" to the flexoelectric effect. 

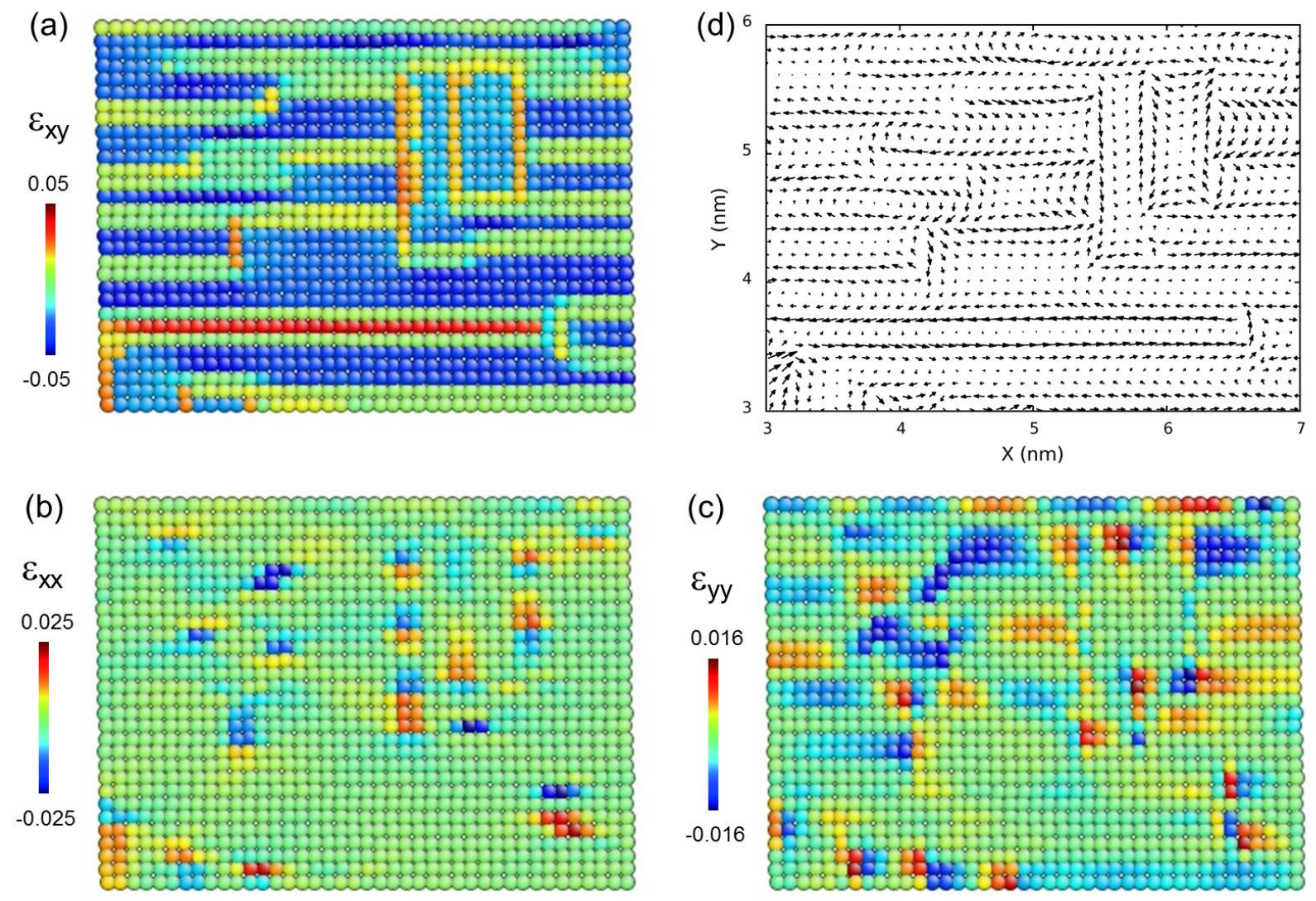

Figure 6 Domain pattern with two orthogonal shear deformations. (a)-(c) atomic images in view of $\varepsilon_{\mathrm{xy}}$, $\varepsilon_{\mathrm{xx}}$ and $\varepsilon_{\mathrm{yy}}$, respectively. (d) dipole configuration. Dipole displacements are amplified by a factor of 25 . The junction density is high leading to extended vortex structures in $P$. 

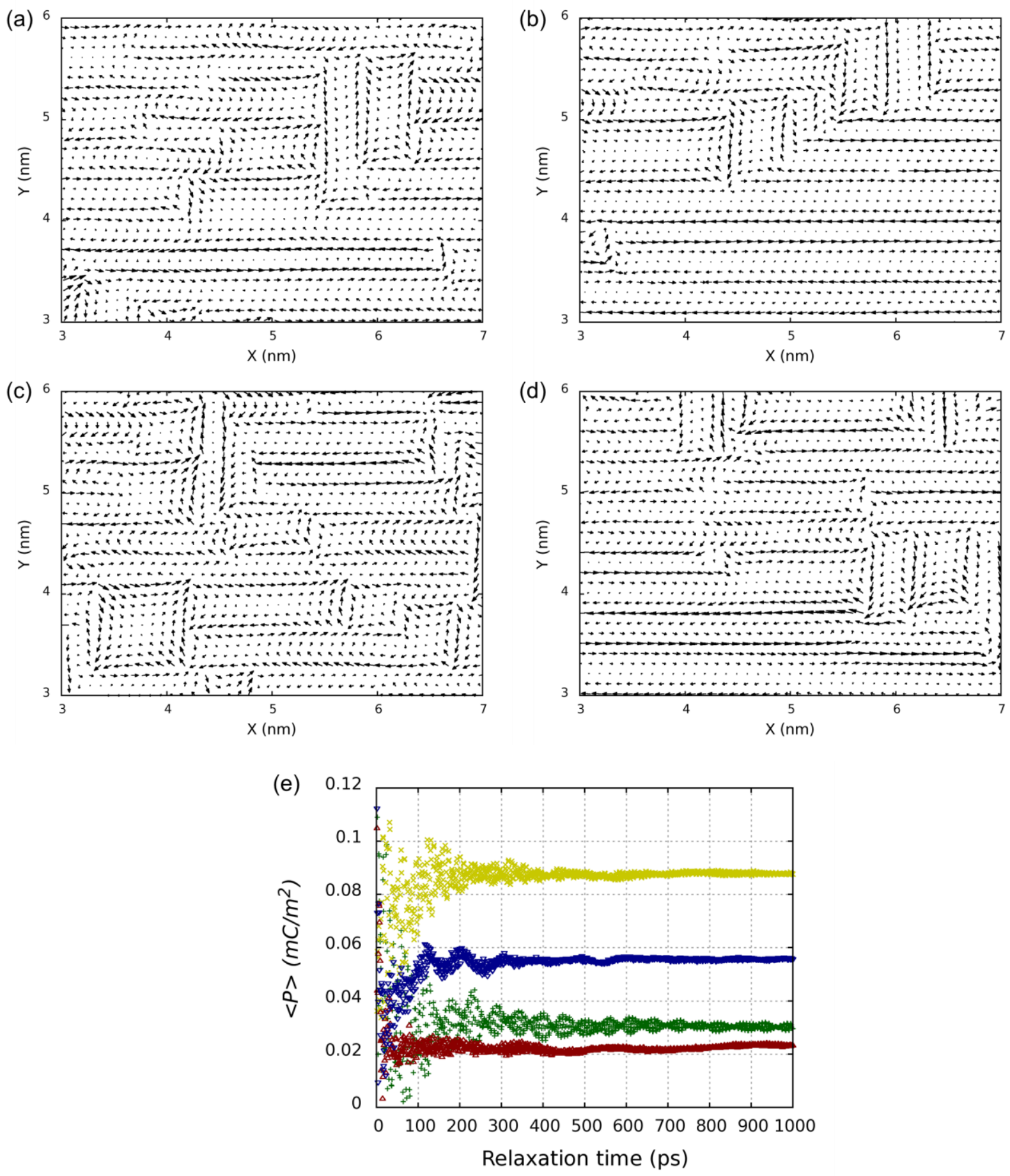

Figure 7 (a)-(d) Four randomly generated complex twin patterns with vortices. Dipole displacements are amplified by a factor of 25 . (e) The ergodic sum over all local polarization densities displays a finite polarization when vortices exist in four randomly generated complex twin patterns. 


\section{Appendix: Determine the parameters in continuum model}

The ferroelasticity energy density is described as

$$
F(Q)=\frac{\mathrm{A}}{2} Q^{2}+\frac{\mathrm{B}}{4} Q^{4}+\frac{g_{\mathrm{Q}}}{2} Q^{\prime 2} .
$$

We used two different models to determine the values of the parameters above. The first one is a uniform shear model. We performed the simple shear to the system and plot the variation of energy with shear strain (see Fig. A1). The data was then fitted in a quadratic-quartic form as $19.8 Q^{2}+8223.5 Q^{4}$. Then we can determine parameters $\mathrm{A}$ and $\mathrm{B}$ in this model as $\mathrm{A}=-39.6$ and $\mathrm{B}=32894.0$.

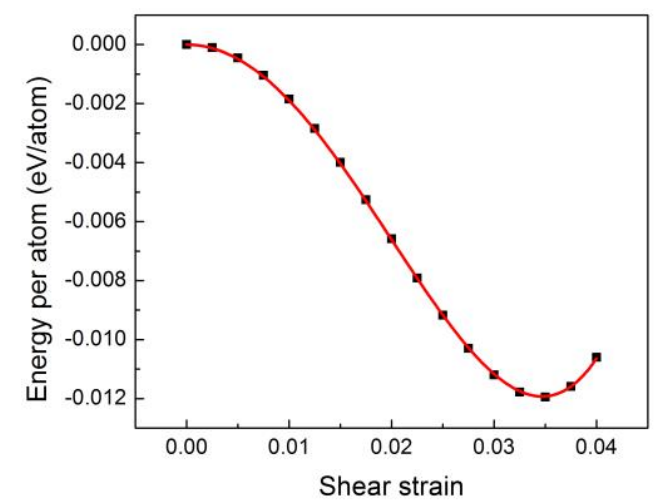

Figure A1 The variation of energy as the function of shear strain in shear model.

The second one is the frozen-phonon model. We created a large box and applied sine-wave displacement as

$u(x)=u_{0} \sin (q x)$,

where $q$ is the wave vector. The strain and strain gradient are then obtained as

$$
Q(x)=u_{0} q \cos (q x)
$$

and

$$
Q(x)^{\prime}=-u_{0} q^{2} \sin (q x) \text {. }
$$

The energy is further calculated to be as the integral of energy density as and finally obtained to be

$$
E\left(q, u_{0}\right)=u_{0}{ }^{2}\left[\frac{1}{4} \mathrm{~A} q^{2}+\left(\frac{3}{32} \mathrm{~B} u_{0}{ }^{2}+\frac{1}{4} g_{\mathrm{Q}}\right) q^{4}\right] \text {. }
$$

We set $u_{0}$ in a serial of values as $0.2,0.015,0.1$ and 0.05 . To determine the parameters, we first fit $E\left(q, u_{0}\right)$ in a quadratic-quartic form as $u_{0}^{2}\left(a q^{2}+b\left(u_{0}\right) q^{4}\right)$. The fitting coefficients of $a$ and $b\left(u_{0}\right)$ were list in the table (see Fig. A2 and Table A1). Then the value of parameter A was obtained to be -40.0 , which is very close to -39.6 that we got in the simple shear model. We then fit $b\left(u_{0}\right)$ in a quadratic form and obtained the values of parameter $\mathrm{B}=33066.7$ (also close to the value in the model above) and $g_{\mathrm{Q}}=5.6$. 

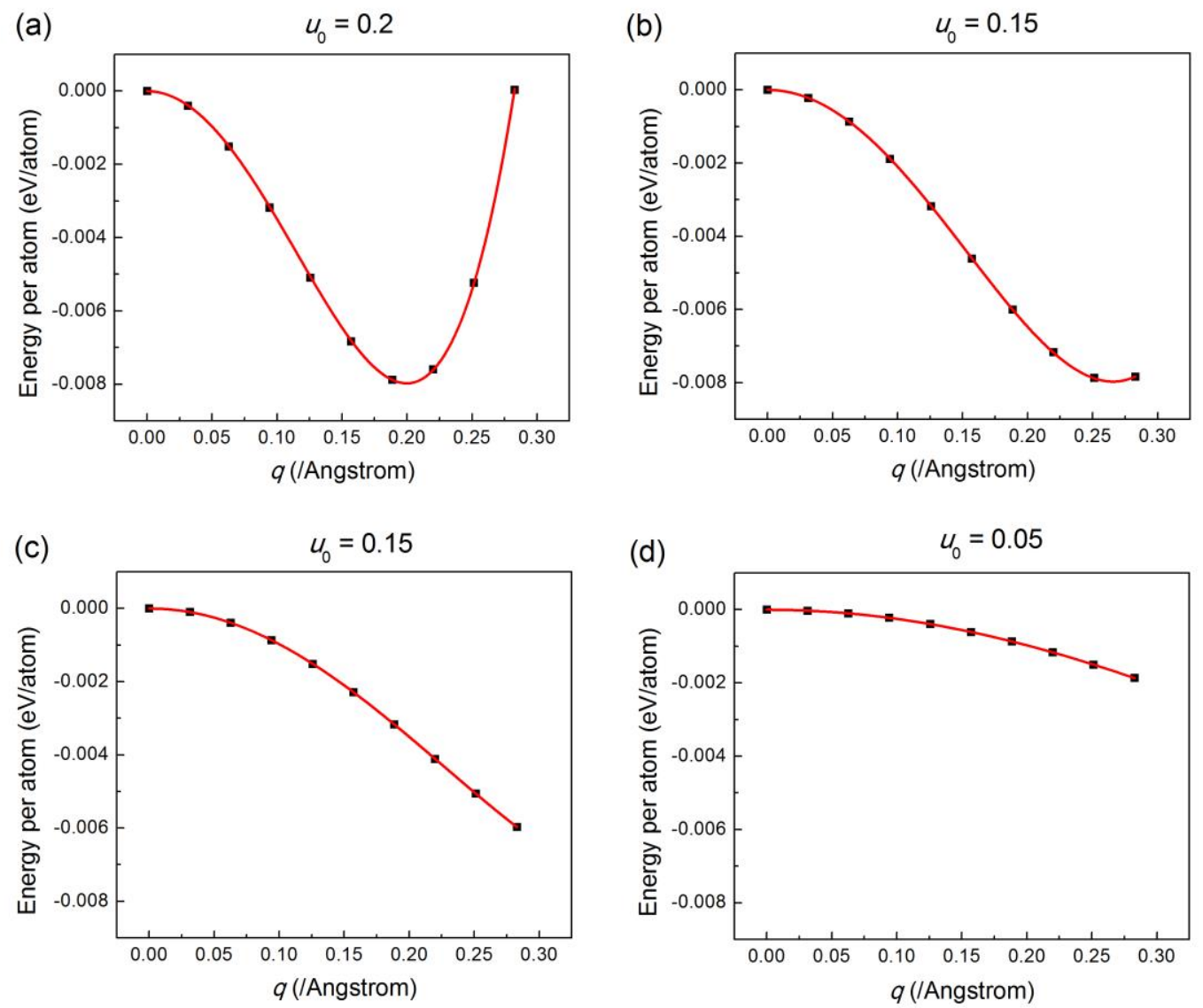

Figure A2 Fitting the data in a quadratic-quartic form with different magnitude of $u_{0}$.

Table A1 The fitting parameters

\begin{tabular}{c|c|c}
\hline$u_{0}$ & $a$ & $b\left(u_{0}\right)$ \\
\hline 0.2 & -10.00 & 125.28 \\
\hline 0.15 & -10.04 & 71.16 \\
\hline 0.1 & -10.00 & 32.30 \\
\hline 0.05 & -10.00 & 9.20 \\
\hline
\end{tabular}

For the flexoelectricity part

$$
G(P)=\frac{\chi^{-1}}{2} P^{2}+\frac{g}{2}\left(P^{\prime}\right)^{2},
$$

we performed the third mode to determine the parameters (see Fig. A3). Specifically, we fixed the A sublattice in cubic shape and only applied frozen-phonon to B sublattice. We obtained the 
data of energy as the function of $q$ and fitted the data in a quadratic form as $0.0068+0.0234 q^{2}$. The inverse susceptibility $\chi^{-1}$ is determined from the zeroth order of $q$ as $\chi^{-1}=0.0134$ $\mathrm{eV} /\left(\mathrm{C} / \mathrm{m}^{2}\right)^{2}$. The parameter $g$ is obtained from the 2 nd order of $q$ as $g=0.0468 \mathrm{eV} /\left(\mathrm{C} / \mathrm{m}^{2} / \AA\right)^{2}$. The coefficient $f$ is then obtained to be $\mu / \chi=0.048 \mathrm{eV} /\left(\mathrm{C} / \mathrm{m}^{2} / \AA\right)$, where $\mu\left(=3.57 \mathrm{C} / \mathrm{m}^{2} / \AA^{-1}\right)$ is the flexoelectricity coefficient.

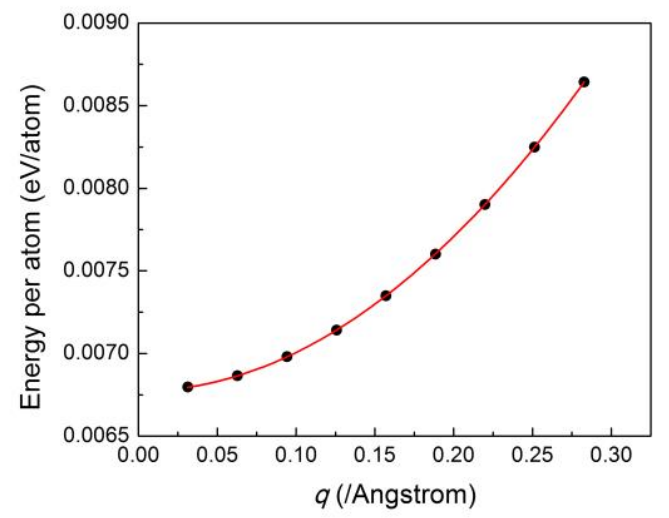

Figure A3 The variation of energy as the function of $q$ in frozen-phonon model. 